\title{
Parametric verification of one-way lithographic wicks
}

\author{
Jijie Zhou ${ }^{* a}$ and Xiao Huang ${ }^{b}$ \\ Received 5th March 2009, Accepted 29th April 2009 \\ First published as an Advance Article on the web 6th May 2009 \\ DOI: $10.1039 / \mathrm{b} 904552 \mathrm{j}$
}

\begin{abstract}
An asymmetric gap was fabricated into a dense array of mediumhigh carbon nanotubes to achieve a one-way fluid transport device through capillary action.
\end{abstract}

Liquid imbibitions through mesopores have been frequently practiced in the field of separation techniques. When we place a liquid drop on a straight or curved strip made of a densely packed array of carbon nanotubes, we can observe spreading of liquid along the strip. Such spreading was demonstrated by both the superficial view of near surface flow and interstitial view of porous imbibition. ${ }^{1}$ This strip of nanotubes was conventionally referred to as a nanowick, because it conveyed liquid along the strip and was featured by site density of carbon nanotubes. The nanotubes of the strip assembled in an array structure as a regular open porous medium. Enormous amounts of information can be carried in nanowicking experiments. The triple line that guided both interstitial wicking and near-surface spreading became significant in many topics, because a free-boundary problem in fluid dynamics was coupled with a nano-interstitial liquid diffusion dilemma. This work focused on a set of geometrical enabling parameters in order to design a diode-like joint on a one-way microfluidic pathway. The geometrical parameters were intended to control the triple line location at the joint.

\section{Principle of operation}

A micrometre sized asymmetric gap, so-named a joint, was fabricated into a wick to hinder fluid transfer process. The void space of the joint was shaped as of an angle bracket, as illustrated in Fig. 1d. Upon optimizing joint configuration, liquid transport at the joint became only nullified from the bulge side to the indented side; in other words, the gap allowed liquid delivery in one direction and blocked the opposite flow. As long as wetting liquid assured a stable ${ }^{2,3}$ flow atop nanotube arrays, joint function would depend upon configuration of both the nanotube array and the joint.

\subsection{Inlet conditions}

An auxiliary structure, liquid reservoirs, was placed at the liquid entrance to a wick. Reservoirs for this work were enclosed and emptied to minimize dead volume of interstitial liquid. These reservoirs enabled convenient input of a liquid drop and kept liquid within the array boundary. Such boundary along flow direction of a nanowick was often considered as nanowick sidewalls. All concave turns

${ }^{a}$ Shanghai Institute of Applied Mathematics and Mechanics, Laboratory of Low-dimensional Carbons and Device Physics, Shanghai University, MC189, 149 Yanchang Road, Shanghai, 200072, People's Republic of China. E-mail: jjzhou@shu.edu.cn; Fax: +862136033287

${ }^{b}$ School of Materials Science and Engineering, Nanyang Technological University, 50 Nanyang Avenue, Singapore, 639798, Singapore of the sidewalls were rounded along flow pathway, as illustrated in Fig. 1a, to prevent liquid from contacting its wafer substrate outside sidewalls.

\subsection{Nanotube array configuration}

Height variation among carbon nanotubes were maintained indifferent from inlet to the joint, because structures such as dam, pit, or inherent-openings would facilitate interstitial wicking rather than surface flow. ${ }^{4}$ We used thermal oxidized silicon wafers as a supporting substrate for nanotube arrays to stand on. When balancing liquid volume from superficial rivulet, near-surface spreading and interstitial wicking (sketched in Fig. 1b), ten-micrometre-high nanotubes appeared optimal for a diode-like wick.

Height, site density, and diameter of the nanotubes were obtained during synthesis by chemical vapor deposition method (First Nano ET2000, CA). The synthesis, taking place at $750{ }^{\circ} \mathrm{C}$ with ethylene as carbon source, resulted in two-dimensional nanotube arrays of a site density in between $10^{11} \mathrm{CNT} \mathrm{cm}^{-2}$ and $10^{12} \mathrm{CNT} \mathrm{cm}^{-2}$. Average tube diameter was around $30-40 \mathrm{~nm}$.

\subsection{Visualization}

Liquid motion on a nanowick was observed under long-distance-lens phase-contrast light microscopy, which enhances contrasts between

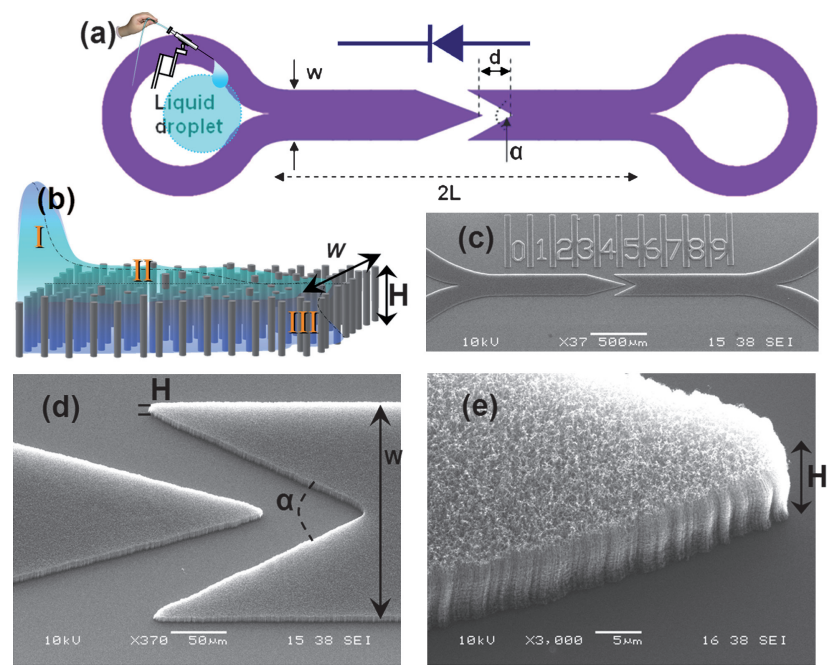

Fig. 1 Nanowick configuration: (a) a schematic diagram showing an asymmetric gap in the middle of a nanowick, where liquid was placed in one of the two liquid reservoirs; (b) sketch of wicking profile ahead of a top rivulet flow with superficial rivulet front $\langle$ I $\rangle$, near-surface spreading $<\mathrm{II}>$, interstitial wicking $<\mathrm{III}>$, and nanotube array (solid cylinders) conceptually illustrated; (c, d) SEM images of a typical diodelike wick, with $\alpha=50^{\circ}$ at the joint; (e) tilted view of a CNT array. 
transparent structures with different refractive index. Diode-like flow was tested in both directions. Flow process was recorded at approximately 30 frames per second (29.95 fps). Detailed nanowick and joint configuration were verified with Scanning Electron Microscopy (SEM, JSM-6360) before and after liquid tests.

\subsection{Joint arrangement}

Geometric parameters of the joint, as well as desired locations of the carbon nanotube arrays, were designed in AutoCAD and transferred onto resist patterns with photolithography techniques. Aluminium underlayer and iron catalyst for growth of carbon nanotubes were deposited sequentially onto the substrate in a magnetron sputtering system. After lift-off with acetone, the catalyst layer revealed the designed locations for nanotubes.

The wick width was set to be either $100 \mu \mathrm{m}$ or $200 \mu \mathrm{m}$. Upstream conducting length $(L)$ was $1 \mathrm{~mm}$, i.e. mainstream flowed $1 \mathrm{~mm}$ to reach the joint. The gap distance $(d)$ at the joint varied from 30 to 100 $\mu \mathrm{m}$; the angle $\alpha$ of the concave corner was designed in the range of $30-60^{\circ}$. Evaporation process ${ }^{5}$ on a microfluidic wick took a longer duration than the required time of conveying liquid. Appropriate values of $d$ and $\alpha$ assured suction of accumulated liquid at the $\alpha$ corner by its projecting counterpart. Despite all experimental complexity, there is no theoretical work to promise a prediction of $d$ and $\alpha$ yet.

\subsection{Liquid on a wick}

The dimensionless capillary number $(\mathrm{Ca}=\mathrm{V \eta} / \gamma)$ characterizes the dynamics of wetting and wicking. At a small scale, molecular dynamic simulations of flow passing carbon nanotube arrays agreed continuum C. W. Oseen's approximation. ${ }^{6}$ Conventionally wicks were referred to as a bundle of cotton, asbestos, or papyrus fibers. Fabricated wicks took advantage of printing technologies for incorporating themselves into microfluidic analysis systems. Progress in nanotechnology made control over geometrical parameters easier, for example using carbon nanotubes as atomically smooth cylindrical structure, and made large-scale integration of nanoscale features practical.

After pore size became smaller than a critical value, the rate of imbibition might not change with surfactant concentration in the feeding solution. ${ }^{7,8}$ Characteristics of flow along a two-dimensional wick were previously studied. Sodium dodecyl sulfate (SDS) aqueous solution at a concentration around the critical micelle concentration (CMC) were selected for liquid to go far. ${ }^{1}$ Therefore, mainstream (a)

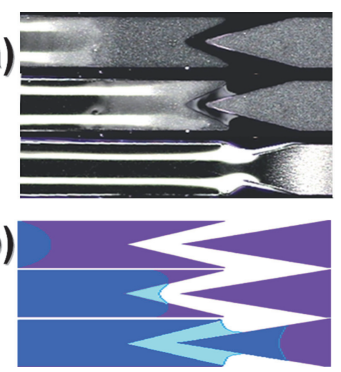

(c)

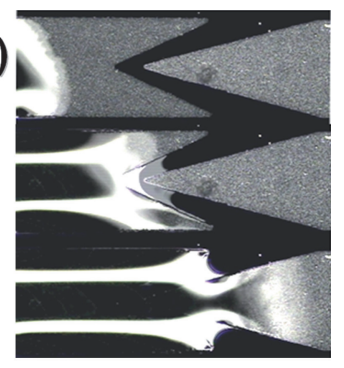

Fig. 2 Three steps of flow at the joint of a diode-like wick: (a) $w=100$ $\mu \mathrm{m}, d=40 \mu \mathrm{m}, \alpha=50^{\circ}$; (b) three schematic steps of wicking as liquid approaching and passing the gap; (c) $w=200 \mu \mathrm{m}, d=60 \mu \mathrm{m}, \alpha=50^{\circ}$. flow on top of nanotubes partially resembled rivulet flow on solid strips, which adapted a parabolic cross-section. ${ }^{9}$ In front of rivulet flow, the nanotube height interfered in liquid-air interface profile; an outline of liquid was sketched as a long-dash-dot line in Fig. 1b. The first frames in Fig. 2a and Fig. 2c demonstrated a superficial view of liquid front before liquid came close to the void space of the joint; the second frames told an instance when liquid jumped over the gap; the third ones exemplified bridging where liquid wicked continuously into the projecting mate.

\section{One-way wicking}

\subsection{Liquid at the joint}

A liquid drop was suspended from a Teflon tip into a nanowick reservoir, and placed close to the wick strip; liquid came into contact with the nanotubes promptly upon its spreading on the substrate. Upon arriving of the rivulet front at the $\alpha$-corner, liquid would conform according to the boundary shape. The indented shape of the $\alpha$-corner built up an intensive capillary pressure. As a consequence, liquid accumulated into the $\alpha$-corner. As soon as an expandable meniscus of the liquid reached any small nanoarray interstices of the other side, liquid continued to convey along the wick. If a sustained meniscus in the $\alpha$-corner reached the projecting counterpart on the other side, liquid would bridge the joint by capillary force as shown in Fig. 2. Otherwise, the combination of corresponding values of $d$ and $\alpha$ were considered as unsuitable to attain the function of a diode-like wick as discussed later in Table 2.

\subsection{Excessive upstream liquid}

When supplied liquid approached the joint from the indented side, liquid visibly accumulated at the $\alpha$-corner. If outreach of liquid came into contact with nanotubes in the counterpart, liquid would jump over the gap and start to flow along. Otherwise, flow became halted at the joint. Therefore, the distance from the $\alpha$-corner to the bulge vertex $(d)$ had to be within a certain value. Once liquid bridged the joint, flow through the gap became smooth, and the gap would not hinder liquid transport in consequent wicking.

For wicks with strip width (w) $200 \mu \mathrm{m}$ and upstream conducting length $(L) 1 \mathrm{~mm}$, the optimum amount of liquid in reservoirs was approximately $0.5 \mu \mathrm{L}$, which was the smallest reading of Hamilton microlitre syringes. If excessive liquid were added into the reservoir to promote liquid outreach, valley flow would develop, i.e. excessive liquid would flow into the V-shaped channel between nanowick sidewalls and the hydrophilic substrate outside nanotube arrays. Valley flow might spread liquid out to nearby structures; as a consequence, addition of valley flow into diode-like wicking would have to reduce packing density of wicks on a wafer. A maximal accumulation of liquid at the $\alpha$-corners was associated with liquid input volume. Liquid reservoir could have been smaller to prevent excessive liquid input. No valley flow should be involved within maxima. We exercised approximately half of maximal volume in functional tests of the wick joint.

Accumulation depth of liquid reached its maximum at approximately $1 \mu \mathrm{L}$ on a $100 \mu \mathrm{m}$ wide wick. The meniscus of maximal accumulation could only be steady when the bulging side of wick stayed out of reach. The accumulation depth, independent of designed gap distance $(d)$, was measured on the medium line of 
Table 1 Maximal accumulation at the $\alpha$-corner

\begin{tabular}{llll}
\hline Wick width $n(w=100 \mu \mathrm{m})$ & Concave angle $(\alpha)$ & Accumulation maximum \\
\hline & $90 \mu \mathrm{m}$ & $62^{\circ}$ & $60 \mu \mathrm{m}$ \\
& $50 \mu \mathrm{m}$ & $47^{\circ}$ & $35 \mu \mathrm{m}$ \\
& $30 \mu \mathrm{m}$ & $35^{\circ}$ & $30 \mu \mathrm{m}$ \\
& & & \\
\hline
\end{tabular}

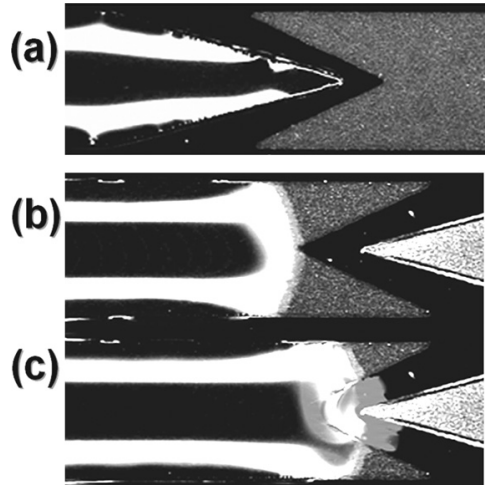

Fig. 3 Flow at the joint of a diode-like wick: (a) blocking of flow; (b, c) bridging the diode-like joint as liquid approaching and passing the gap, when the bulging half of wick was soaked.

Table 2 Thresholds of $\alpha$ and $\mathrm{d}$ for $13 \mu$ m-high wicks

\begin{tabular}{llll}
\hline$\Delta d=10 \mu \mathrm{m}$ & \multicolumn{3}{l}{$\Delta \alpha=10^{\circ}$} \\
\hline$w=100 \mu \mathrm{m}$ & $w=100 \mu \mathrm{m}$ & $w=200 \mu \mathrm{m}$ & $w=200 \mu \mathrm{m}$ \\
$d=40 \mu \mathrm{m}$ & $d=50 \mu \mathrm{m}$ & $d=70 \mu \mathrm{m}$ & $d=70 \mu \mathrm{m}$ \\
$\alpha=50^{\circ}$ & $\alpha=50^{\circ}$ & $\alpha=50^{\circ}$ & $\alpha=60^{\circ}$ \\
Functional diode & Non-functional & Functional diode & Non-functional \\
\hline
\end{tabular}

$\alpha$-corners as given in Table 1 . Tall nanotubes $(H)$ lead to larger depth of accumulation.

\subsection{Functional values}

When we increased the gap distance at the joint in Fig. 2a from $40 \mu \mathrm{m}$ to $50 \mu \mathrm{m}$, liquid accumulated up to a depth of $42 \mu \mathrm{m}$ at the concave corner, and was not able to bridge over. Therefore, a gap distance of $20-40 \mu \mathrm{m}$ was considered as suitable to $50^{\circ} \alpha$-corners for a $13 \mu \mathrm{m}$ high and $100 \mu \mathrm{m}$ wide wick. This gap distance was chosen to be larger than $20 \mu \mathrm{m}$ in order to block liquid flow in the other direction on the wick materials. The roughness of nanowick sidewalls had to be taken into account for robust blocking as the one shown in Fig. 3a. Diodelike flow over the joint when liquid approached from both directions was not systematically investigated, because our wick did not form a multi-loop circuit to make synchronization possible. In Fig. 3b, liquid from the right side stopped at the gap. In Fig. 3c, the right-side nanotubes remained to be soaked as liquid on the left continuously approached and bridged the joint.
Proper design of the joint on a microfluidic pathway enabled liquid to pass in only one direction, like electron flow in diodes. With all liquid restricted within the wick width during the whole transfer process, there exists a set of threshold values of geometrical parameters (Table 2). Within the range, the joint functions like a diode for fluidic transport; beyond, the joint blocks liquid flow in both directions. Introducing logic concept into microfluidic systems could facilitate lab-on-a-chip automation.

\section{Conclusion}

Microfluidic devices significantly minimize reagent volume and cost. Development and optimization of such fluidic devices involve surface forces, diffusion efficiency, and hydrodynamics. We have introduced a diode-like one-way valve on a microfluidic wick. By designing an asymmetric gap on the wicking pathway, liquid was conveyed in one direction and became blocked at the gap in the other direction. Suitable values to attain the diode-like function were investigated. Corresponding geometrical parameters, as well as thresholds for proper design, were exemplified. A one-way wick provides a novel way of fluidic manipulation, and could be integrated in capillary pumping loops, on-chip fluid circuits, and micro total analysis system.

This work is supported by the National Science Foundation of China (10802045), Shanghai Leading Academic Discipline Project (Y0103), and Academic Research Fund (RG27/05) from Ministry of Education, Singapore.

\section{Notes and references}

1 J. J. Zhou, F. Noca and M. Gharib, Nanotechnology, 2006, 17, 48454853.

2 R. Lenormand, J. Phys.: Condens. Matter, 1990, 2, SA79-SA88.

3 M. Dube, M. Rost, K. R. Elder, M. Alava, S. Majaniemi and T. Ala-Nissila, Phys. Rev. Lett., 1999, 83, 1628-1631.

4 X. Huang, J. J. Zhou, E. Sansom, M. Gharib and C. H. Sow, Nanotechnology, 2007, 18, 305301.

5 G. Guena, C. Poulard and A. M. Cazabat, J. Colloid Interface Sci, 2007, 312, 164-171.

6 J. H. Walther, T. Werder, R. L. Jaffe and P. Koumoutsakos, Phys. Rev. E, 2004, 69, art. no.-062201.

7 V. M. Starov, S. A. Zhdanov and M. G. Velarde, J. Colloid Interface Sci., 2004, 273, 589-595.

8 V. Dutschk, K. G. Sabbatovskiy, M. Stolz, K. Grundke and V. M. Rudoy, J. Colloid Interface Sci., 2003, 267, 456-462.

9 A. A. Darhuber, S. M. Troian and W. W. Reisner, Phys. Rev. E, 2001, 6403, art. no.-031603. 